\title{
Systematic review of basket trials, umbrella trials, and platform trials: a landscape analysis of master protocols
}

Jay J. H Park ${ }^{1,2}$, Ellie Siden², Michael J. Zoratti ${ }^{3}$, Louis Dron², Ofir Harari', Joel Singer ${ }^{4,5}$, Richard T. Lester ${ }^{1}$, Kristian Thorlund ${ }^{2,3,6}$ and Edward J. Mills $s^{2,3,6^{*}}$

\begin{abstract}
Background: Master protocols, classified as basket trials, umbrella trials, and platform trials, are novel designs that investigate multiple hypotheses through concurrent sub-studies (e.g., multiple treatments or populations or that allow adding/removing arms during the trial), offering enhanced efficiency and a more ethical approach to trial evaluation. Despite the many advantages of these designs, they are infrequently used.
\end{abstract}

Methods: We conducted a landscape analysis of master protocols using a systematic literature search to determine what trials have been conducted and proposed for an overall goal of improving the literacy in this emerging concept. On July 8, 2019, English-language studies were identified from MEDLINE, EMBASE, and CENTRAL databases and hand searches of published reviews and registries.

Results: We identified 83 master protocols (49 basket, 18 umbrella, and 16 platform trials). The number of master protocols has increased rapidly over the last five years. Most have been conducted in the US ( $n=44 / 83)$ and investigated experimental drugs $(n=82 / 83)$ in the field of oncology $(n=76 / 83)$. The majority of basket trials were exploratory (i.e., phase $\mathrm{I} / \mathrm{I} ; n=47 / 49)$ and not randomized $(n=44 / 49)$, and more than half $(n=28 / 48)$ investigated only a single intervention. The median sample size of basket trials was 205 participants (interquartile range, Q3-Q1 [IQR]: 500-90 = 410), and the median study duration was 22.3 (IQR: 74.1-42.9 = 31.1) months. Similar to basket trials, most umbrella trials were exploratory $(n=16 / 18)$, but the use of randomization was more common $(n=8 / 18)$. The median sample size of umbrella trials was 346 participants (IQR: $565-252=313$ ), and the median study duration was 60.9 (IQR: 81.3-46.9=34.4) months. The median number of interventions investigated in umbrella trials was $5(\mathrm{IQR}: 6-4=2)$. The majority of platform trials were randomized $(n=15 / 16)$, and phase III investigation ( $n=7 / 15$; one did not report information on phase) was more common in platform trials with four of them using seamless II/III design. The median sample size was 892 (IQR: 1835-255 $=1580$ ), and the median study duration was 58.9 (IQR: 101.3-36.9 = 64.4) months.

Conclusions: We anticipate that the number of master protocols will continue to increase at a rapid pace over the upcoming decades. More efforts to improve awareness and training are needed to apply these innovative trial design methods to fields outside of oncology.

Keywords: Master protocols, Basket trials, Umbrella trials, Platform trials, Multi-arm, multi-stage design

\footnotetext{
* Correspondence: emills@mteksciences.com

${ }^{2}$ MTEK Sciences, 802-777 West Broadway, Vancouver, BC V5Z 1J5, Canada

${ }^{3}$ Department of Health Research Methods, Evidence, and Impact, McMaster

University Medical Centre, 1280 Main Street West, 2C Area, Hamilton, ON L8S

4K1, Canada

Full list of author information is available at the end of the article
}

(c) The Author(s). 2019 Open Access This article is distributed under the terms of the Creative Commons Attribution 4.0 International License (http://creativecommons.org/licenses/by/4.0/), which permits unrestricted use, distribution, and reproduction in any medium, provided you give appropriate credit to the original author(s) and the source, provide a link to the Creative Commons license, and indicate if changes were made. The Creative Commons Public Domain Dedication waiver (http://creativecommons.org/publicdomain/zero/1.0/) applies to the data made available in this article, unless otherwise stated. 


\section{Background}

Advancements in genomics, particularly in tumor sequencing, have improved our ability to differentiate cancers by their genetic mutations [1]. This has fueled the efforts towards "precision oncology", in which therapies are selected to specifically target cancers on the basis of their genetic mutations. These innovative treatments are commonly referred to as targeted therapies [2]. However, it is unrealistic to investigate the broad spectrum of genetic sub-populations by conventional trial designs. Thus, "master protocol" frameworks have been proposed to provide a means of comprehensively and adaptively evaluating treatments from the field of oncology [3, 4].

The term "master protocol" refers to a single overarching design developed to evaluate multiple hypotheses, and the general goals are improving efficiency and establishing uniformity through standardization of procedures in the development and evaluation of different interventions $[5,6]$. Under a common infrastructure, the master protocol may be differentiated into multiple parallel substudies to include standardized trial operational structures, patient recruitment and selection, data collection, analysis, and management [3-6].

Master protocols are often classified into "basket trials", "umbrella trials", and "platform trials" [3-6]. Basket trials refer to designs in which a targeted therapy is evaluated on multiple diseases that have common molecular alternations. Umbrella trials, on the other hand, evaluate multiple targeted therapies for a single disease that is stratified into subgroups by molecular alternation. Basket trials and umbrella trials employ a molecular screening protocol that allows either recruitment of different diseases with the common molecular alteration(s) or that differentiates the single disease into different molecular subtypes. Platform trials, also referred to as multi-arm, multi-stage (MAMS) design trials [7-10], are trials that evaluate several interventions against a common control group and can be perpetual $[3,5,11,12]$. This design has pre-specified adaptation rules to allow dropping of ineffective intervention(s) and flexibility of adding new intervention(s) during the trial $[3,5,11,12]$.

Master protocols may be tailored and adapted to suit the research objectives of multiple clinical indications, but master protocols have not been well established in fields outside of oncology $[4,13]$. There may be missed opportunities in research fields outside of oncology. Thus, improved understanding and awareness of these research designs are important for the research community. Methodological summaries of master protocols to date have not been comprehensive, and a cursory review of the literature returned no systematic literature reviews. With the intent of improving literacy in this emerging field, we conducted this comprehensive systematic literature review as a landscape analysis of master protocols.

\section{Methods}

This systematic literature review was designed in accordance with the Preferred Reporting Items for Systematic Reviews and Meta-Analysis (PRISMA) guidelines [14] EQUATOR checklist for this review is provided in the Supplementary (Additional file 2).

\section{Data sources and searches}

Systematic searches were conducted on July 8, 2019, in MEDLINE, EMBASE, and the Cochrane Central Register of Controlled Trials. As no validated literature search strategy has been published, our strategies were developed on the basis of a review of key papers, including the Draft Guidance of the US Food and Drug Administration (FDA) [3-6, 15]. We complemented the search terms of "master protocols", "basket trials", "umbrella trials", and "platform trials" with several search terms specific to "adaptive trial designs" to improve the sensitivity of our search. The search strategies for each database are presented in Additional file 1: Tables S1-S3. We supplemented our database searches with a review of bibliographies from included publications. In addition, we searched trial registries (ClinicalTrials.gov and ISRCTN registry) for registered master protocols. Search terms used for ClinicalTrials.gov are reported in Additional file 1: Table S4. The list of published reviews related to master protocols that we reviewed is provided in Additional file 1: Table S5.

\section{Study inclusion and exclusion criteria}

Complete study eligibility is described in Table 1 . In brief, we included peer-reviewed publications, conference abstracts, and clinical registry records reporting on master protocols (basket trials, umbrella trials, and platform trials) that have been proposed, are ongoing, or have already been conducted. We defined "basket trials" as any prospective clinical trials that investigated the utility (e.g., effectiveness, dosage, and safety) of intervention(s) in a study population of multiple diseases with common predictive biomarkers or other common predictive patient characteristics that can be used to predict whether a patient will respond to a specific intervention (or both) as the unifying eligibility criteria. We defined "umbrella trials" as any prospective clinical trials that investigated the utility of targeted interventions based on predictive biomarkers or other patient characteristics or both. In umbrella trials, the single disease population (e.g., single histology cancer) is stratified into multiple subgroups on predictive biomarkers or other characteristics or both. We defined "platform trials" as any clinical trials that allowed for the intervention $\operatorname{arm}(\mathrm{s})$ to be 
Table 1 PICOS (population, intervention, comparator, outcomes, study design) criteria

\begin{tabular}{|c|c|}
\hline Category & Inclusion criteria \\
\hline Population & Humans \\
\hline Interventions & No restrictions \\
\hline Comparator & No restrictions \\
\hline Outcomes & No restrictions \\
\hline $\begin{array}{l}\text { Study } \\
\text { design }\end{array}$ & $\begin{array}{l}\text { Master protocols were defined as a single overarching protocol that has been designed to be divided into multiple sub-studies that } \\
\text { could allow for evaluation of multiple interventional hypotheses. These included: } \\
\text { - Basket trials } \\
\text { - Umbrella trials } \\
\text { - Platform trials }\end{array}$ \\
\hline Other & Peer-reviewed publications and conference abstracts with results or published protocols in the English language \\
\hline
\end{tabular}

'Basket trials' were defined as any prospective clinical trials that tested the utility (e.g., effectiveness, dosage, and safety) of intervention(s) in a study population of multiple diseases with common predictive biomarkers and/or other common predictive patient characteristics that can be used to predict whether a patient will respond to a specific intervention as the unifying eligibility criteria

'Umbrella trials' were defined as any prospective clinical trials that tested the utility of targeted interventions based on predictive biomarkers or other patient characteristics or both; in umbrella trials, the single disease study population is stratified into multiple subgroups on predictive biomarkers or other characteristics or both

'Platform trials' were defined as any clinical trials that allowed the intervention arm(s) to be dropped and the flexibility of introducing new intervention(s) during the trial. Platform trials are sometimes referred to as multi-arm, multi-stage (MAMS) designs, but the MAMS designs that do not allow flexibility of adding new arms during the trial are not truly platform trials

dropped and the flexibility of introducing new intervention(s) during the trial. Graphical displays of basket trials, umbrella trials, and platform trials are provided in Fig. 1. We excluded non-English language studies.

Two reviewers (JJHP and MJZ) independently reviewed all abstracts and proceedings identified in the literature searches. The full-text publications of potentially relevant abstracts were then retrieved and assessed for eligibility. Two reviewers also screened the bibliographies of published literature reviews on master protocols (JJHP and ES) and trial registries (JJHP and LD). Discrepancies in study selection were resolved by discussion or, when necessary, by a third investigator (KT or EJM).

\section{Data extraction}

Study design elements, patient characteristics, and outcomes were extracted independently by two investigators (JJHP and ES) using a standardized, piloted data extraction form. We recorded information on trial registry, trial recruitment status, phase, randomization, masking, number of clinical centers, sample size, trial duration, interventions and control, disease area, age of population, number of conventional diseases recruited, key eligibility for stratification, number of subgroups defined, and geographic location of the master protocols. Discrepancies were resolved by discussion.

\section{Data synthesis}

A meta-analysis was not conducted for this study, and we present the findings of this landscape analysis descriptively. We report on temporal trends of master protocols, geographical representation, and trial and disease characteristics of each of the three master protocols (basket trials, umbrella trials, and platform trials).

\section{Role of the funding source}

This study was not funded.

\section{Results \\ Literature search}

The study selection process is presented in Additional file 1: Figure S1. We identified 5869 abstracts from our database searches, and 140 more records were identified through hand searches of bibliographies and trial registries. Of these, 639 records were selected for full-text review. In total, 214 publications describing 83 trials met our inclusion criteria. Thirty-four trials were available only through trial registries, and three trials were in the pre-recruitment phase (NCT03339843, NCT03915678, and NCT03872427). A complete list of trials and the corresponding citations is provided in Additional file 1: Tables S6-S8. In summary, we identified 49 basket trials, 18 umbrella trials, and 16 platform trials.

\section{Trends of master protocols}

There has been a rapid increase in the number of master protocols published in the last five years (Fig. 2). From our literature search, we identified nine completed and published master protocol trials, including results. The first master protocol conducted was a basket trial called the Imatinib Target Exploration Consortium Study B2225 [16, 17], which started in 2001. This was followed by the platform trial STAMPEDE, which was first proposed in $2005[8,9$, 18-28]. We identified 68 ongoing master protocols (39 basket trials, 17 umbrella trials, and 12 platform trials) recruiting patients; of these, 11 basket trials [29-38], eight umbrella trials [39-46], and four 


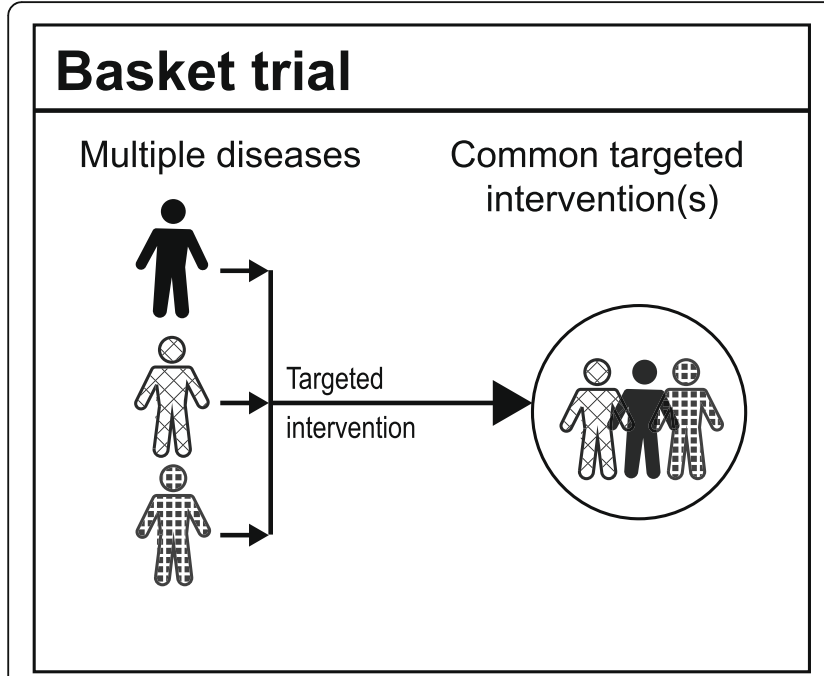

\section{Umbrella trial}

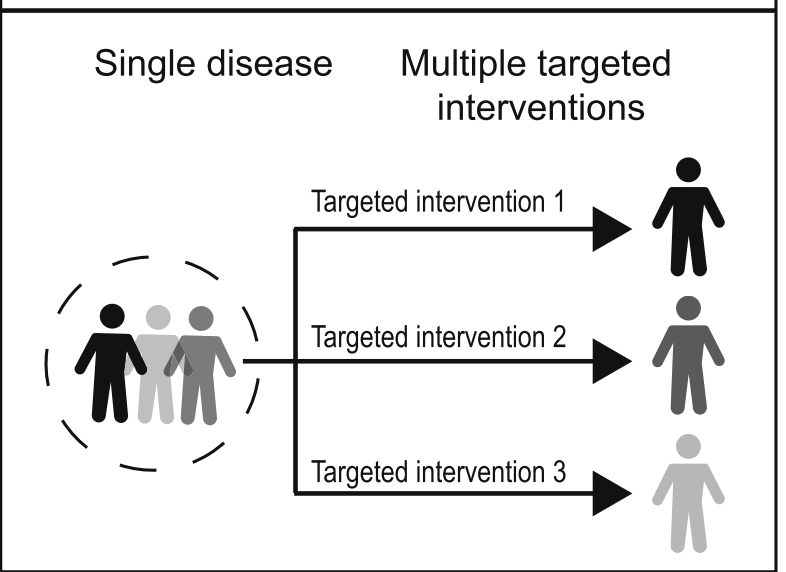

\section{Platform trial}

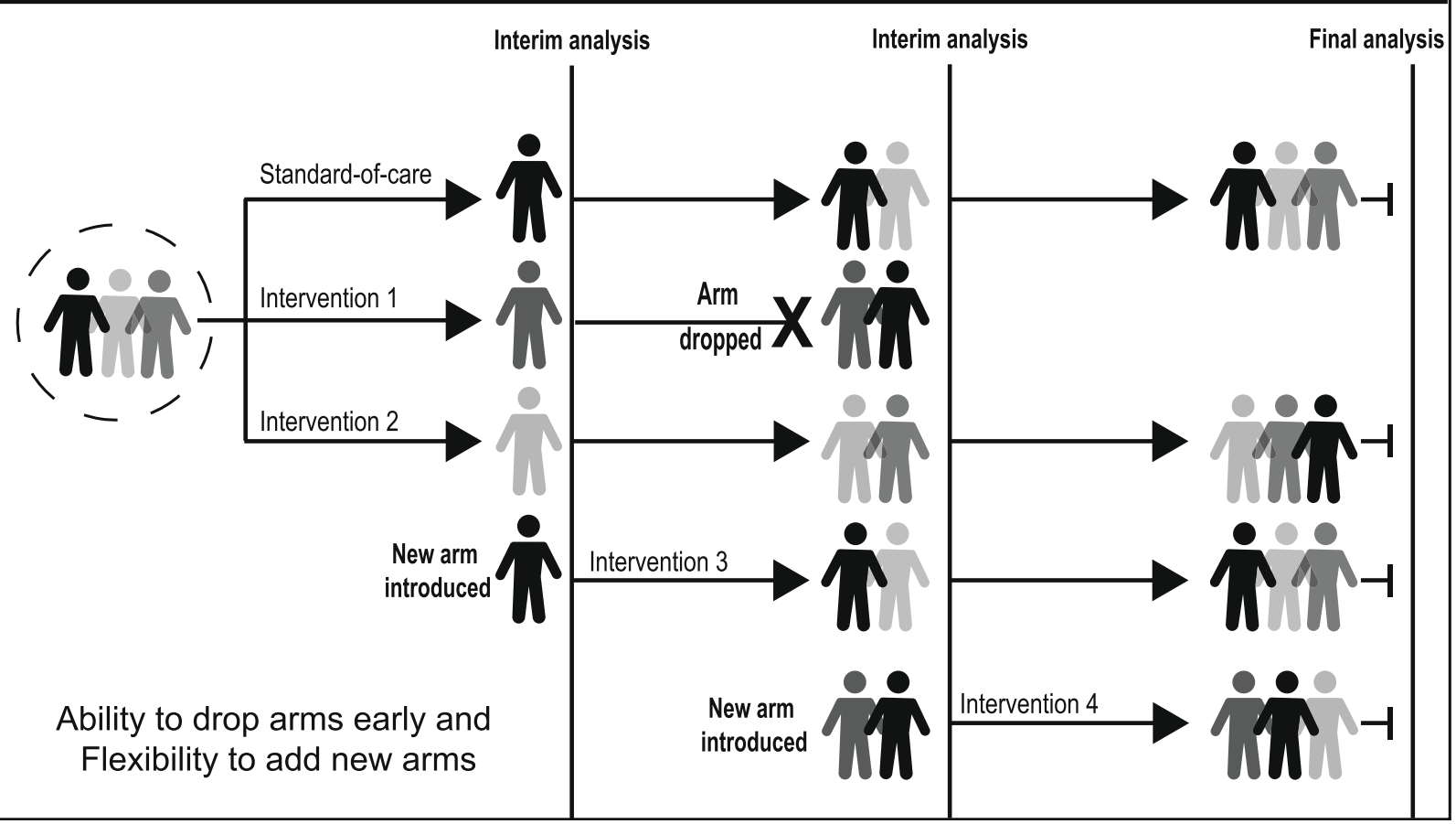

Fig. 1 Graphical representation of basket trials, umbrella trials, and platform trials. This figure illustrates a simple graphical representation of basket, umbrella, and platform trials. There may be other forms of master protocols. The clip art in the figure was generated by the authors

platform trials $[24,47-50]$ have published results (Additional file 1: Table S10).

At the time of writing (August 1, 2019), one platform trial (LEAP; NCT03092674) is suspended for an unscheduled safety data review [51]. EBOLA (NCT02380 625), a platform trial supported by the Bill \& Melinda Gates Foundation in response to the 2014 West Africa Ebola outbreak, has been terminated, as it could not be launched in time in response to the outbreak [52].
Trial characteristics of master protocols

Trial characteristics of the master protocols are presented in Additional file 1: Table S9, and the sample size distribution of these master protocols displayed as box plots is provided in Fig. 3.

The majority of master protocols were basket designs, and 49 are identified in the current review. Among basket trials, all but one involved a drug investigation ( $n=$ 48/49); NCT03003195 was the exception as a proposed 


\section{Number of Master Protocols over Time: \\ Basket Trials, Umbrella Trials, and Platform Trials}

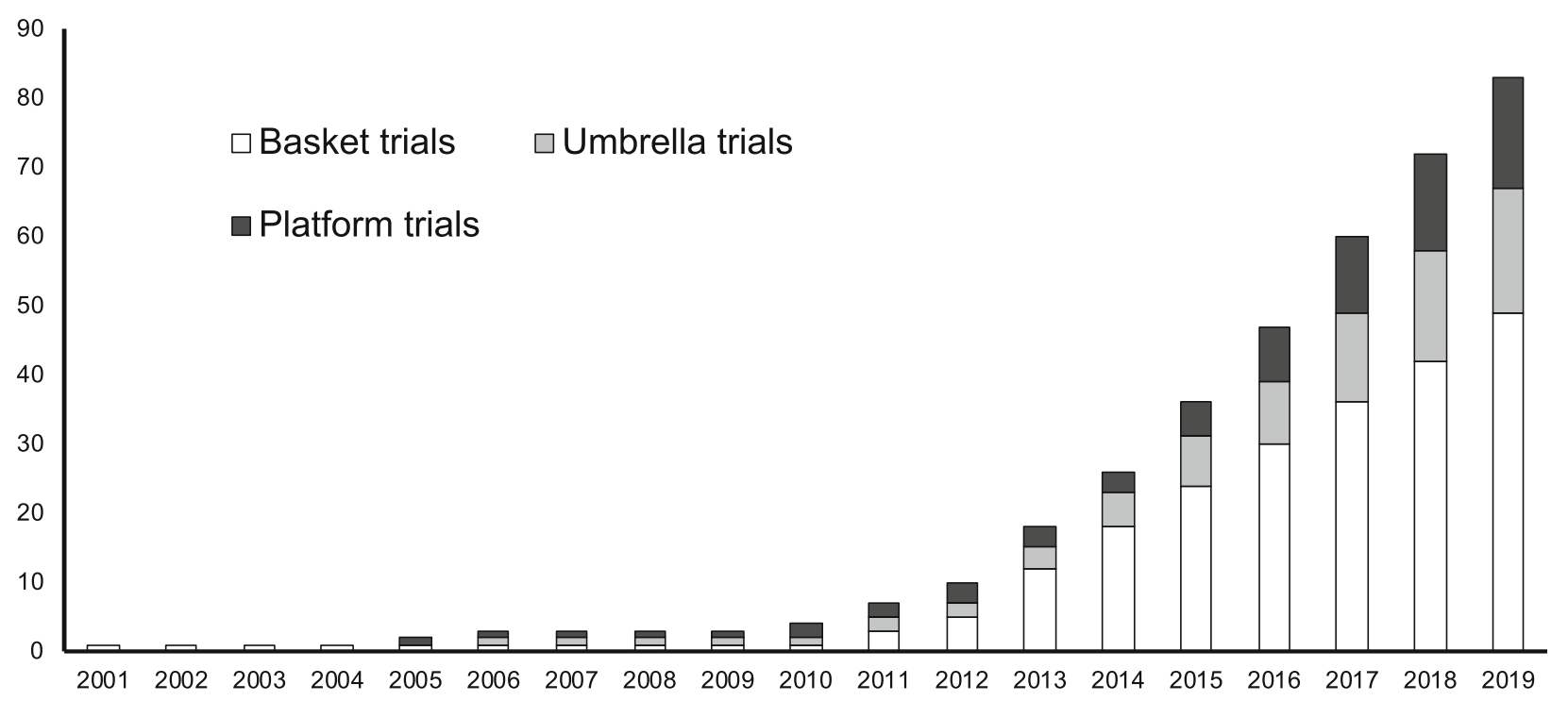

Fig. 2 Trends of master protocols over time. This figure illustrates the accumulating number of basket (white), umbrella (gray), and platform (black) trials over time. The clip art in the figure was generated by the authors

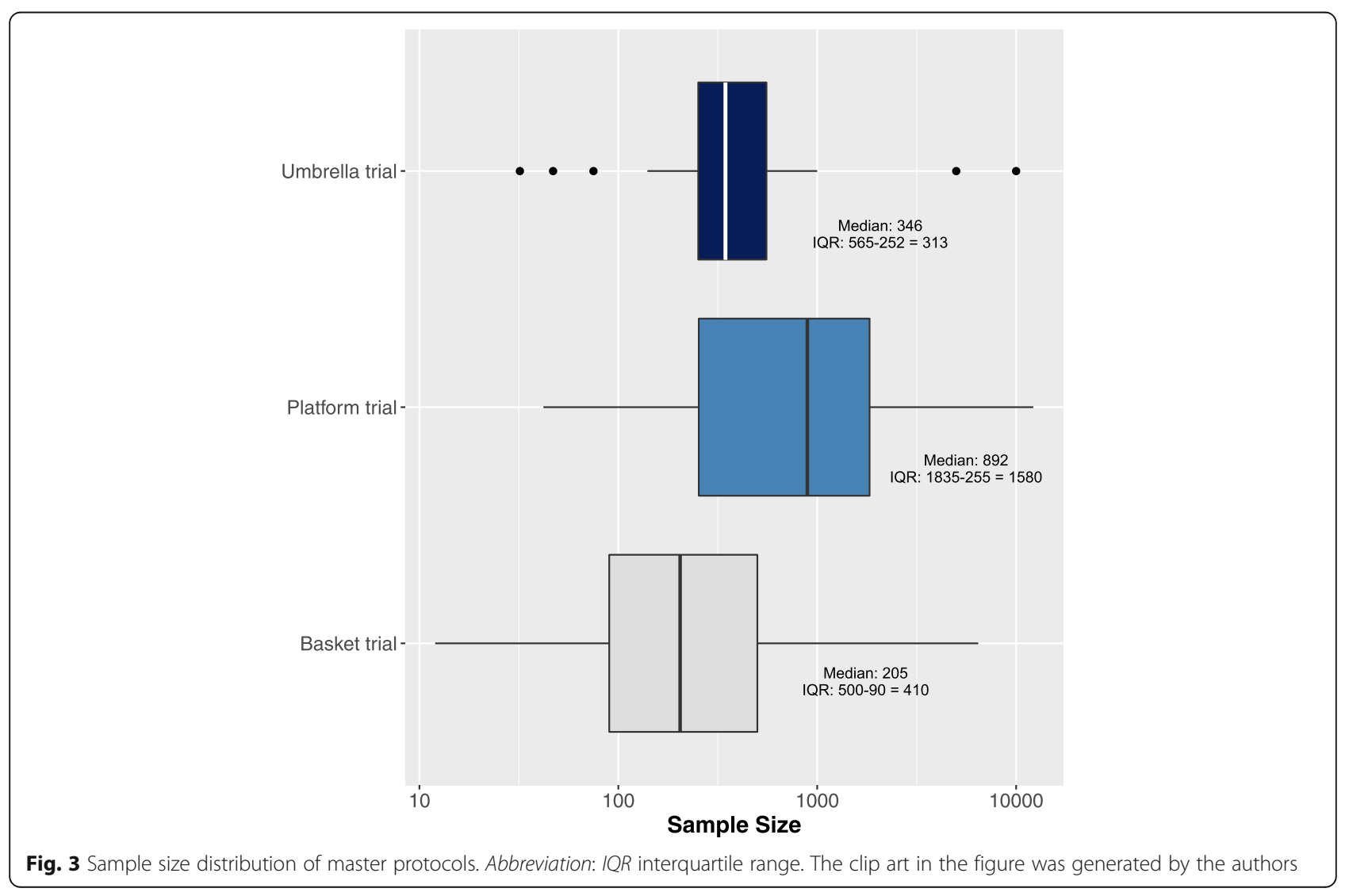


vaccine basket trial. The majority of basket trials were exploratory (i.e., phase I or II; $n=47 / 49$ ) and were open-label $(n=46 / 49)$; more than half of the included basket trials investigated only a single intervention arm ( $n=28 / 48$; one did not report information on the number of interventions), and the majority did not involve a control group or randomization $(n=44 / 49)$. The median sample size of basket trials was 205 participants (interquartile range, Q3-Q1 [IQR]: 500-90 = 410), and the median study duration was 22.3 (IQR: 74.1-42.9 = 31.1) months. ALCHEMIST (NCT02193282, NCT02595944, and NCT02201992) and CLUSTER (NCT02059291) [53-55] were the only phase III basket trials, which were comprised of three interventions arms and were of an open-label design.

Eighteen umbrella trials were identified. All umbrella trials investigated experimental drugs, and eight out of the 18 trials used randomization to assign patients to different arms. The median sample size of umbrella trials was 346 participants (IQR: $565-252=313$ ), and the median study duration was 60.9 (IQR: $81.3-46.9=34.4$ ) months. The median number of interventions investigated in umbrella trials was 5 (IQR: $6-4=2$ ). Similar to basket trials, the majority of umbrella trials were exploratory $(n=16 / 18)$ and open-label $(n=16 / 17$; one did not report information on blinding).

Our review returned 16 platform trials. All of the platform trials involved investigation of experimental drugs. The median sample size was 892 (IQR: 1835-255 = 1580 ), and the median study duration was 58.9 (IQR: $101.3-36.9=64.4)$ months. Nearly all platform trials were of open-label design $(n=12 / 14$; two trials did not report information on blinding), similar to basket and umbrella trials. However, phase III investigation was more common among platform trials $(n=7 / 15$; one did not report information on phase) in contrast to basket and umbrella trials; four of these seven platform trials were seamless II/III trials. In the majority of platform trials, patients were assigned by randomization $(n=15 /$ 16). PRISM (NCT03527147) was the only non-randomized platform trial, although this is currently a phase I study. However, the trial registry of PRISM indicates that future arms may be added. In STAMPEDE $[8,9$, 18-28] and I-SPY2 [49, 50, 56-61], several agents have graduated from the phase II evaluation with seamless transitions into phase III evaluations. The phase III evaluation for the I-SPY program is called I-SPY3.

\section{Disease characteristics of master protocols}

The patient and disease characteristics of master protocols are provided in Additional file 1: Table S10. Most studies were in adult populations $(n=69 / 83)$, and nearly all were in the field of oncology $(n=76 / 83)$. No umbrella trials were conducted outside of oncology. Notably, two basket trials were conducted for other clinical indications, namely hereditary periodic fevers (CLUSTER; NCT02059291) [53-55] and complement-mediated disorders (TNT0009 Basket trial). Additionally, five platform trials have been designed for influenza (ALIC4E; ISRCTN27908921) [62], Ebola (EBOLA) [52], pneumonia (REMAP-CAP; NCT027 35707), pre-operative surgery (UPMC REMAP; NCT0 3861767), and Alzheimer's disease (The DIAN-TU platform; NCT01760005) [48].

\section{Geographic representation of master protocols}

The information on the geographical representation of the current master protocols is provided in Additional file 1: Table S11. The majority of current master protocols have taken place in the US $(n=44 / 83)$ (Fig. 4). Other high-income countries such as the UK $(n=25)$, France $(n=23)$, Spain $(n=17)$, and Canada $(n=13)$ were the next most common countries. There were no master protocols observed from low-income countries, although the EBOLA (NCT02380625) trial had been proposed for Guinea, Sierra Leone, and Liberia [52]. Two upper-middle-income countries, Brazil and Mexico, were involved in the DIAN-TU platform trial (NCT01 760005), but these countries accounted for only three of 36 study sites [48]. China, an upper-middle-income country, has centers participating in FUTURE (NCT03 805399), GBM AGILE [47, 56, 63], TRUMP (NCT0 3574402), and VE-BASKET (NCT01524978) $[64,65]$ trials, but it should be noted that China accounts for only a minority of study sites in GBM AGILE and VEBASKET.

\section{Discussion}

To the best of our knowledge, this is the first landscape analysis of master protocols. This was achieved through a methodologically robust and rigorous systematic literature review that included queries of medical literature databases, reference lists of included studies, and clinical trial registries. Unlike previous publications on master protocols that were limited in scope to select only specific studies, this review catalogues all master protocols that have been conducted or proposed to date. Of the 83 master protocols ( 49 basket trials, 18 umbrella trials, and 16 platform trials), the majority have involved investigation of experimental drugs in adult patients for the field of oncology.

Our study may have been limited by variability of terminology and lack of standardized nomenclatures and indexing of master protocols in the medical databases. However, we believe that this was offset by our rigorous approach that had strong supplemental searching strategies inclusive of several search terms on adaptive trial designs. We first reviewed the key papers on master protocols to gain an overview of the existing literature [3-6, 

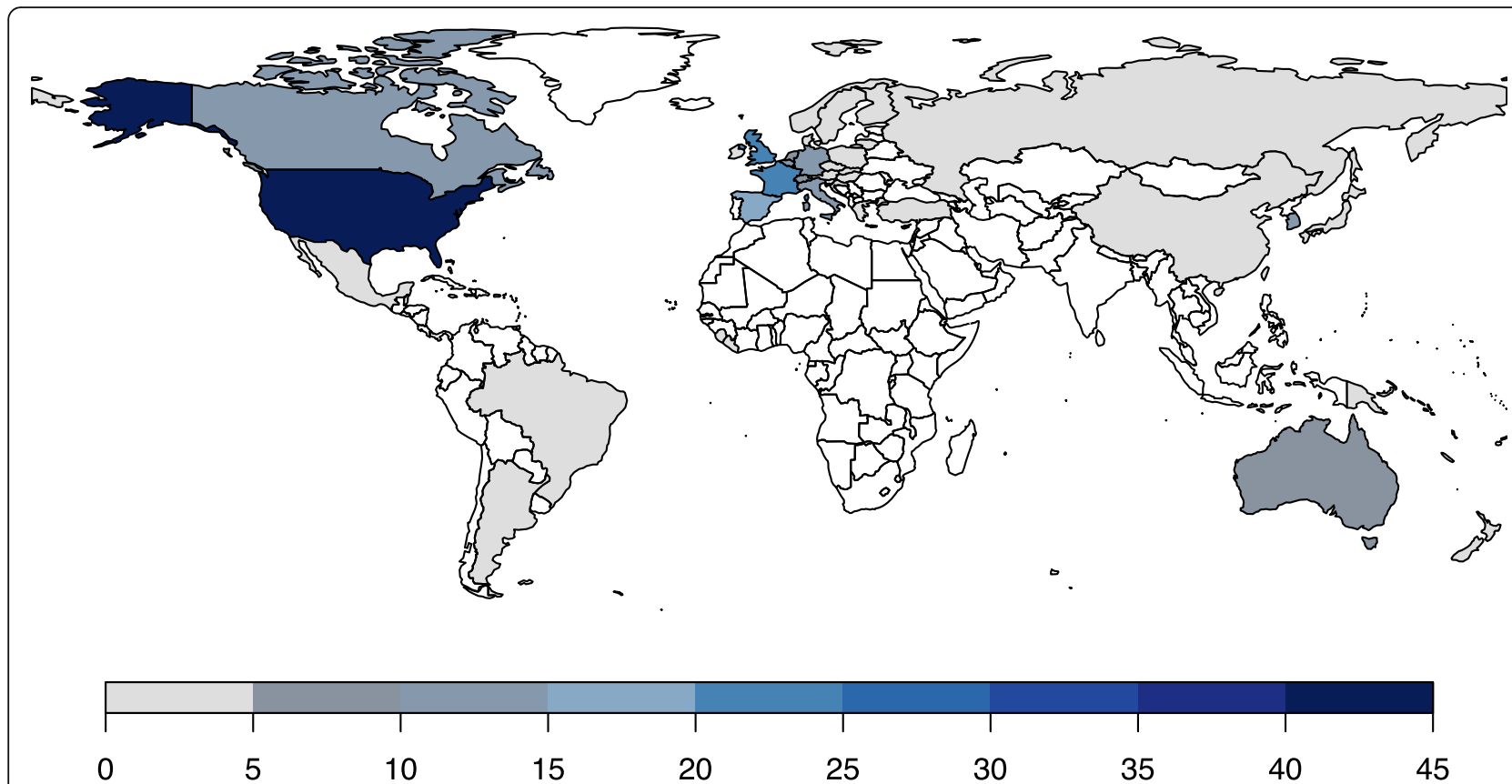

Fig. 4 Geographical representation of master protocols. This figure illustrates the accumulating number of basket (white), umbrella (gray), and platform (black) trials over time. The clip art in the figure was generated by the authors

15] before coming up with our search strategy. (We recommend that readers review these key publications.) Then developed search terms were complemented by hand searches of bibliographies of 52 published reviews that we found before and during the screening process (Additional file 1: Table S5) and international trial registries.

We have identified several directions for future research. An improved approach to standardized nomenclature and database indexing is essential to improve the identification and retrieval of these study designs. Moreover, efforts are needed to improve the awareness and technical expertise $[3-6,15]$ of master protocols to investigators in fields outside of oncology and in geographic regions outside of high-income countries (e.g., the US). Platform trials, by nature, are potentially perpetual and permit research questions to evolve over time in the context of new information [11, 12]. Basket trials and umbrella trials have had considerable emphasis and dependencies on the accuracy of genomic biomarkers used to characterize cancers, in addition to their histology and location [5], but it is important to point out that other baseline patient characteristics may be used to determine the intervention strategies. Thus, an emphasis on the study of how genomic screening tests impact the operational characteristics of these biomarker trials is warranted. Comparing different nomenclatures used in published trials and reviews may also be warranted in order to come up with a consensus on master protocols.

\section{Conclusion}

This is the first systematic review-based bibliometric analysis of master protocols. The number of master protocols, especially in the last five years, has increased dramatically and we anticipate that this trend will continue over the coming years. Master protocols, particularly platform trials, have the potential to improve the efficiency across the broad spectrum of clinical trial research. This study was carried out at an opportunistic time, as the FDA released draft guidance on master protocols in September 2018 [15]. We anticipate that this landscape analysis may be useful for regulatory agencies as well as clinical investigators and readers who are looking to broaden their expertise in this emerging field.

\section{Additional files}

Additional file 1: Supplementary Appendix. Supplementary to "Systematic review of basket trials, umbrella trials, and platform trials: A landscape analysis of master protocols". (DOCX 604 kb)

Additional file 2: EQUATOR Checklist. EQUATOR Checklist for "Systematic review of basket trials, umbrella trials, and platform trials: A landscape analysis of master protocols". (DOC $63 \mathrm{~kb}$ )

Abbreviations

FDA: US Food and Drug Administration; IQR: Interquartile range

Acknowledgments

Not applicable. 


\section{Authors' contributions}

JJHP conceived and designed the study. JJHP, ES, MJZ, and LD acquired the data. JJHP, ES, MJZ, LD, OH, JS, RTL, KT, and EJM analyzed and interpreted the data. JJHP, ES, MJZ, LD, and OH drafted the manuscript. KT and EJM obtained funding. JS, RTL, KT, and EJM provided administrative, technical, or material support. JS, RTL, KT, and EJM supervised the study. All authors critically revised the manuscript for important intellectual content and read and approved the final manuscript.

\section{Funding}

This study was not funded.

\section{Availability of data and materials}

All data generated or analyzed during this study are included in this published article.

\section{Ethics approval and consent to participate}

Not applicable.

\section{Consent for publication}

Not applicable.

\section{Competing interests}

The authors declare that they have no competing interests.

\section{Author details}

${ }^{1}$ Experimental Medicine, Department of Medicine, 10th Floor, 2775 Laurel Street, Vancouver, BC V5Z 1M9, Canada. ${ }^{2}$ MTEK Sciences, 802-777 West Broadway, Vancouver, BC V5Z 1J5, Canada. ${ }^{3}$ Department of Health Research Methods, Evidence, and Impact, McMaster University Medical Centre, 1280 Main Street West, 2C Area, Hamilton, ON L8S 4K1, Canada. ${ }^{4}$ School of Population and Public Health, University of British Columbia, 2206 E Mall, Vancouver, BC V6T 1Z3, Canada. ${ }^{5}$ Data and Methodology Program, CIHR Canadian HIV Trials Network, 588 - 1081 Burrard Street, Vancouver, BC V6Z 1Y6, Canada. ${ }^{6}$ Knowledge Integration, Bill and Melinda Gates Foundation, 500 5th Ave N, Seattle, WA 98109, USA.

\section{Received: 11 April 2019 Accepted: 19 August 2019}

Published online: 18 September 2019

\section{References}

1. Kumar-Sinha C, Chinnaiyan AM. Precision oncology in the age of integrative genomics. Nat Biotechnol. 2018;36:46-60.

2. Ke X, Shen L. Molecular targeted therapy of cancer: The progress and future prospect. Front Lab Med. 2017;1:69-75.

3. Redman MW, Allegra CJ. The Master Protocol Concept. Semin Oncol. 2015; 42:724-30.

4. Hirakawa A, Asano J, Sato H, Teramukai S. Master protocol trials in oncology: Review and new trial designs. Contemp Clin Trials Commun. 2018;12:1-8.

5. Renfro LA, Sargent DJ. Statistical controversies in clinical research: basket trials, umbrella trials, and other master protocols: a review and examples. Ann Oncol. 2017;28:34-43.

6. Woodcock J, LaVange LM. Master protocols to study multiple therapies, multiple diseases, or both. N Engl J Med. 2017;377:62-70.

7. Parmar MK, Sydes MR, Cafferty FH, Choodari-Oskooei B, Langley RE, Brown $L$, et al. Testing many treatments within a single protocol over 10 years at MRC Clinical Trials Unit at UCL: Multi-arm, multi-stage platform, umbrella and basket protocols. Clin Trials. 2017;14:451-61.

8. Sydes MR, Parmar MKB, James ND, Clarke NW, Dearnaley DP, Mason MD, et al. Issues in applying multi-arm multi-stage methodology to a clinical trial in prostate cancer: the MRC STAMPEDE trial. Trials. 2009;10:39.

9. Sydes MR, Spears MR, Mason MD, Clarke NW, Dearnaley DP, de Bono JS, et al. Adding abiraterone or docetaxel to long-term hormone therapy for prostate cancer: Directly randomised data from the STAMPEDE multi-arm, multi-stage platform protocol. Ann Oncol. 2018;29:1235-48.

10. Parker CC, James ND, Brawley CD, Clarke NW, Hoyle AP, Ali A, et al. Radiotherapy to the primary tumour for newly diagnosed, metastatic prostate cancer (STAMPEDE): a randomised controlled phase 3 trial. Lancet. 2018:392:2353-66.

11. Berry SM, Connor JT, Lewis RJ. The platform trial: an efficient strategy for evaluating multiple treatments. JAMA. 2015;313:1619-20.
12. Saville BR, Berry SM. Efficiencies of platform clinical trials: A vision of the future. Clin Trials. 2016;13:358-66.

13. Lam VK, Papadimitrakopoulou V. Master protocols in lung cancer: experience from Lung Master Protocol. Curr Opin Oncol. 2018;30:92-7.

14. Moher D, Liberati A, Tetzlaff J, Altman DG. Preferred reporting items for systematic reviews and meta-analyses: the PRISMA statement. Ann Intern Med. 2009;151:264-9.

15. Master Protocols. Efficient Clinical Trial Design Strategies to Expedite Development of Oncology Drugs and Biologics Guidance for Industry (Draft Guidance) [https://www.fda.gov/downloads/Drugs/

GuidanceComplianceRegulatorylnformation/Guidances/UCM621817.pdf]. Accessed 1 Oct 2018.

16. Heinrich MC, Joensuu H, Demetri GD, Corless CL, Apperley J, Fletcher JA, et al. Phase II, open-label study evaluating the activity of imatinib in treating life-threatening malignancies known to be associated with imatinibsensitive tyrosine kinases. Clin Cancer Res. 2008;14:2717-25.

17. McArthur GA, Demetri GD, van Oosterom A, Heinrich MC, Debiec-Rychter M, Corless $\mathrm{CL}$, et al. Molecular and clinical analysis of locally advanced dermatofibrosarcoma protuberans treated with imatinib: Imatinib Target Exploration Consortium Study B2225. J Clin Oncol. 2005;23:866-73.

18. James ND, Sydes MR, Clarke NW, Mason MD, Dearnaley DP, Anderson J, et al. Systemic therapy for advancing or metastatic prostate cancer (STAMPEDE): a multi-arm, multistage randomized controlled trial. BJU Int. 2009;103:464-9

19. Sydes MR, Parmar MKB, Mason MD, Clarke NW, Amos C, Anderson J, et al. Flexible trial design in practice - stopping arms for lack-of-benefit and adding research arms mid-trial in STAMPEDE: a multi-arm multi-stage randomized controlled trial. Trials. 2012;13:168.

20. Gilson C, Chowdhury S, Parmar MKB, Sydes MR, Investigators S. Incorporating Biomarker Stratification into STAMPEDE: an Adaptive Multiarm, Multi-stage Trial Platform. Clin Oncol (Royal College of Radiologists (Great Britain)). 2017;29:778-86.

21. James N, De Bono J, Spears MR, Clarke NW, Mason MD, Dearnaley D, et al. Adding abiraterone for patients (pts) with high-risk prostate cancer (PCa) starting long-term androgen deprivation therapy (ADT): Outcomes in nonmetastatic (M0) patients from STAMPEDE (NCT00268476). Ann Oncol. 2017; 28:v620.

22. James ND, De Bono JS, Spears MR, Clarke N, Mason MD, Dearnaley DP, et al. Adding abiraterone for men with high-risk prostate cancer (PCa) starting long-term androgen deprivation therapy (ADT): Survival results from STAMPEDE (NCT00268476). J Clin Oncol. 2017;35:18_suppl, LBA5003LBA5003. https://ascopubs.org/action/showCitFormats?doi=10.1200/JCO.201 7.35.18_suppl.LBA5003.

23. James ND, de Bono JS, Spears MR, Clarke NW, Mason MD, Dearnaley DP, et al. Abiraterone for Prostate Cancer Not Previously Treated with Hormone Therapy. N Engl J Med. 2017;377:338-51.

24. James ND, Sydes MR, Mason MD, Clarke NW, Anderson J, Dearnaley DP, et al. Celecoxib plus hormone therapy versus hormone therapy alone for hormone-sensitive prostate cancer: first results from the STAMPEDE multiarm, multistage, randomised controlled trial. Lancet Oncol. 2012;13: $549-58$.

25. James ND, Sydes MR, Mason MD, Clarke NW, Dearnaley DP, Dwyer J, et al. Celecoxib plus hormone therapy vs hormone therapy alone for hormonesensitive prostate cancer: First results from the STAMPEDE randomised controlled trial (MRC PR08). Eur J Cancer. 2011;47:11.

26. Mason MD, Clarke NW, James ND, Dearnaley DP, Spears MR, Ritchie AW, et al. Adding Celecoxib With or Without Zoledronic Acid for HormoneNaive Prostate Cancer: long-Term Survival Results From an Adaptive, Multiarm, Multistage, Platform, Randomized Controlled Trial. J Clin Oncol. 2017:35:1530.

27. Sydes MR, James ND, Mason MD, Clarke NW, Amos C, Anderson J, et al. Flexible trial design in practice - Dropping and adding arms in STAMPEDE: A multi-arm multi-stage randomised controlled trial. Trials, 2011;12(Suppl 1):A3.

28. Sydes MR, Mason MD, Spears MR, Clarke NW, Dearnaley D, Ritchie AWS, et al. PR Adding abiraterone acetate plus prednisolone (AAP) or docetaxel for patients (pts) with high-risk prostate cancer (PCa) starting long-term androgen deprivation therapy (ADT): Directly randomised data from STAMPEDE (NCT00268476). Ann Oncol. 2017;28:v619.

29. Lopez-Chavez A, Thomas A, Rajan A, Raffeld M, Morrow B, Kelly R, et al. Molecular profiling and targeted therapy for advanced thoracic 
malignancies: a biomarker-derived, multiarm, multihistology phase II basket trial. J Clin Oncol. 2015;33:1000-7.

30. Hainsworth JD, Meric-Bernstam F, Swanton C, Hurwitz H, Spigel DR, Sweeney C, et al. Targeted Therapy for Advanced Solid Tumors on the Basis of Molecular Profiles: Results From MyPathway, an Open-Label, Phase lla Multiple Basket Study. J Clin Oncol. 2018;36:536-42.

31. Li BT, Shen R, Buonocore D, Olah ZT, Ni A, Ginsberg MS, et al. Adotrastuzumab emtansine in patients with HER2 mutant lung cancers: Results from a phase II basket trial. J Clin Oncol. 2017;35:15_suppl, 8510-8510. https://ascopubs.org/action/showCitFormats?doi=10.1200/JCO.2017.35.15_ suppl.8510.

32. Aletaha D, Alasti F, Smolen JS. Disease activity states of the DAPSA, a psoriatic arthritis specific instrument, are valid against functional status and structural progression. Ann Rheum Dis. 2017;76:418-21.

33. Muhlbacher J, Jilma B, Wahrmann M, Bartko J, Eskandary F, Schorgenhofer C, et al. Blockade of HLA Antibody-Triggered Classical Complement Activation in Sera From Subjects Dosed With the Anti-C1s Monoclonal Antibody TNT009-Results from a Randomized First-in-Human Phase 1 Trial. Transplantation. 2017;101:2410-8.

34. Hyman D, Piha-Paul S, Saura C, Arteaga C, Mayer I, Shapiro G, et al. Neratinib + fulvestrant in ERBB2-mutant, HER2-non-amplified, estrogen receptor (Ex positive, metastatic breast cancer (MBC): Preliminary analysis from the phase II SUMMIT trial). Cancer Res. 2017;77(4 Suppl):Abstract nr PD2-08. https:// cancerres.aacrjournals.org/content/77/4_Supplement/PD2-08.

35. Perez JMT, Leary A, Besse B, Ponce DCS, Arrondeau J, Moreno V, et al. Efficacy and safety of lurbinectedin (PM1183, Zepsyre ${ }^{\oplus}$ ) in small cell lung cancer (SCLC): results from a phase 2 study. J Clin Oncol. 2018;36:15_suppl, 8570-8570. https://ascopubs.org/action/showCitFormats?doi=10.1200/JCO.2 018.36.15_suppl.8570.

36. Bardia A, Mayer IA, Vahdat LT, Tolaney SM, Isakoff SJ, Diamond JR, et al. Sacituzumab Govitecan-hziy in Refractory Metastatic Triple-Negative Breast Cancer. N Engl J Med. 2019;380:741-51.

37. Schöffski P, Sufliarsky J, Gelderblom H, Blay J-Y, Strauss SJ, Stacchiotti S, et al. Crizotinib in patients with advanced, inoperable inflammatory myofibroblastic tumours with and without anaplastic lymphoma kinase gene alterations (European Organisation for Research and Treatment of Cancer 90101 CREATE): a multicentre, single-drug, prospective, nonrandomised phase 2 trial. Lancet Respir Med. 2018;6:431-41.

38. Chen A, Conley B, Hamilton S, Williams M, O'Dwyer P, Arteaga C, et al. NClMolecular Analysis for Therapy Choice (NCI-MATCH) trial: a novel publicprivate partnership. Eur J Cancer. 2016;69:S137.

39. Kim ES, Herbst RS, Wistuba II, Lee JJ, Blumenschein GR Jr, Tsao A, et al. The BATTLE trial: personalizing therapy for lung cancer. Cancer Discov. 2011;1:44-53.

40. Papadimitrakopoulou V, Lee JJ, Wistuba II, Tsao AS, Fossella FV, Kalhor N, et al. The BATTLE-2 Study: A Biomarker-Integrated Targeted Therapy Study in Previously Treated Patients With Advanced Non-Small-Cell Lung Cancer. J Clin Oncol. 2016;34:3638-47.

41. Spigel DR, Reynolds C, Waterhouse D, Garon EB, Chandler J, Babu S, et al. Phase 1/2 Study of the Safety and Tolerability of Nivolumab Plus Crizotinib for the First-Line Treatment of Anaplastic Lymphoma Kinase Translocation Positive Advanced Non-Small Cell Lung Cancer (CheckMate 370). J Thorac Oncol. 2018;13:682-8.

42. LoRusso PM, Boerner SA, Pilat MJ, Forman KM, Zuccaro CY, Kiefer JA, et al. Pilot Trial of Selecting Molecularly Guided Therapy for Patients with NonV600 BRAF-Mutant Metastatic Melanoma: Experience of the SU2C/MRA Melanoma Dream Team. Mol Cancer Ther. 2015;14:1962-71.

43. Herbst RS, Gandara DR, Hirsch FR, Redman MW, LeBlanc M, Mack PC, et al. Lung Master Protocol (Lung-MAP)-A Biomarker-Driven Protocol for Accelerating Development of Therapies for Squamous Cell Lung Cancer: SWOG S1400. Clin Cancer Res. 2015;21:1514-24.

44. Wick W, Dettmer S, Berberich A, Kessler T, Karapanagiotou-Schenkel I, Wick A, et al. N2M2 (NOA-20) phase I/II trial of molecularly matched targeted therapies plus radiotherapy in patients with newly diagnosed non-MGMT hypermethylated glioblastoma. Neuro-Oncology. 2019;21:95-105.

45. Turner N, Bye H, Kernaghan S, Proszek P, Fribbens C, Moretti L, et al. Abstract OT1-06-03: The plasmaMATCH trial: A multiple parallel cohort, open-label, multi-centre phase II clinical trial of ctDNA screening to direct targeted therapies in patients with advanced breast cancer (CRUK/15/010). San Antonio: AACR; 2018.

46. Graham JS, Valle JW, Evans J, Grose DB, Paul J, Stobo J, et al. PRIMUS-001: An adaptive phase II study of FOLFOX-A (FOLFOX and nab-paclitaxel) versus
AG (nab-paclitaxel and gemcitabine) in patients with metastatic pancreatic cancer, with integrated biomarker evaluation (ISRCTN75002153) - Part of Precision-Panc. J of Clin Oncol. 2018 36:15_suppl, TPS4158-TPS4158. https:// ascopubs.org/action/showCitFormats?doi=10.1200/JCO.2018.36.15_suppl. TPS4158.

47. Alexander BM, Ba S, Berger MS, Berry DA, Cavenee WK, Chang SM, et al. Adaptive Global Innovative Learning Environment for Glioblastoma: GBM AGILE. Clin Cancer Res. 2018;24:737-43.

48. Bateman RJ, Benzinger TL, Berry S, Clifford DB, Duggan C, Fagan AM, et al. The DIAN-TU Next Generation Alzheimer's prevention trial: Adaptive design and disease progression model. Alzheimers Dement. 2017;13:8-19.

49. Park JW, Liu MC, Yee D, Yau C, van't Veer LJ, Symmans WF, et al. Adaptive Randomization of Neratinib in Early Breast Cancer. N Engl J Med. 2016;375:11-22.

50. Rugo HS, Olopade Ol, DeMichele A, Yau C, van't Veer LJ, Buxton MB, et al. Adaptive Randomization of Veliparib-Carboplatin Treatment in Breast Cancer. N Engl J Med. 2016;375:23-34.

51. Walter RB, Michaelis LC, Othus M, Uy GL, Radich JP, Little RF, et al. Intergroup LEAP trial (S1612): A randomized phase 2/3 platform trial to test novel therapeutics in medically less fit older adults with acute myeloid leukemia. Am J Hematol. 2018;93:E49-52.

52. Berry SM, Petzold EA, Dull P, Thielman NM, Cunningham CK, Corey GR, et al. A response adaptive randomization platform trial for efficient evaluation of Ebola virus treatments: A model for pandemic response. Clin Trials (London, England). 2016;13:22-30.

53. De Benedetti F, Anton J, Gattorno M, Lachmann H, Kone-Paut I, Ozen S, et al. Efficacy and safety of Canakinumab in patients with periodic fever syndromes (colchicine-resistant fmf, hids/mkd and traps): Results from a phase 3, pivotal, umbrella trial. Pediatr Rheumatol. 2017;15(Suppl 1):01. https://www.ncbi.nlm.nih.gov/pmc/articles/PMC5461530/.

54. De Benedetti F, Anton J, Gattorno M, Lachmann H, Kone-Paut I, Ozen S, et al. Pharmacokinetics and pharmacodynamics of canakinumab in patients with periodic fever syndromes (colchicine-resistant FMF, HIDS/MKD and TRAPS): Results from a phase III pivotal umbrella trial. Pediatr Rheumatol. 2017;15(Suppl 1):P20. https://www.ncbi.nlm.nih.gov/pmc/articles/ PMC5461530\%.

55. De Benedetti F, Gattorno M, Anton J, Ben-Chetrit E, Frenkel J, Hoffman HM, et al. Canakinumab for the Treatment of Autoinflammatory Recurrent Fever Syndromes. N Engl J Med. 2018;378:1908-19.

56. Berry DA, Graves T, Connor J, Alexander B, Cloughesy T, Barker A, et al. Adaptively randomized seamless-phase multiarm platform trial: Glioblastoma Multiforme Adaptive Global Innovative Learning Environment (GBM AGILE). Cancer Res. 2017;77(13 Suppl):Abstract nr 3594. https://doi. org/10.1158/1538-7445.AM2017-3594. https://cancerres.aacrjournals.org/ content/77/13_Supplement/3594.

57. Barker AD, Sigman CC, Kelloff GJ, Hylton NM, Berry DA, Esserman LJ. I-SPY 2: an adaptive breast cancer trial design in the setting of neoadjuvant chemotherapy. Clin Pharmacol Ther. 2009;86:97-100.

58. Forero A, Yee D, Buxton MB, Symmans WF, Chien AJ, Boughey JC, et al. Efficacy of Hsp90 inhibitor ganetespib plus standard neoadjuvant therapy in high-risk breast cancer: Results from the I-SPY 2 trial. Cancer Res. 2017;77(4 Suppl):Abstract nr P6-11-02. https://cancerres.aacrjournals.org/content/77/4_ Supplement/P6-11-02.

59. Nanda R, Liu MC, Yau C, Asare S, Hylton N, Van't Veer L, et al. Pembrolizumab plus standard neoadjuvant therapy for high-risk breast cancer (BC): Results from I-SPY 2. J Clin Oncol. 2017;35:15_suppl, 506-506. https://ascopubs.org/action/showCitFormats?doi=10.1200/JCO.2017.35.15_ suppl.506.

60. Paoloni M, Lyandres J, Buxton MB, Berry DA, Esserman L, DeMichele A, et al. A longitudinal look at toxicity management within a platform trial: Lessons from the I-SPY 2 TRIAL. Cancer Res. 2017;77(4 Suppl):Abstract nr P211-02. https://cancerres.aacrjournals.org/content/77/4_Supplement/P2-11-02.

61. Yee D, Paoloni M, Van't Veer L, Sanil A, Yau C, Forero A, et al. The evaluation of ganitumab/metformin plus standard neoadjuvant therapy in high-risk breast cancer: Results from the ISPY 2 trial. Cancer Res. 2017;77(4 Suppl): Abstract nr P6-11-04. https://cancerres.aacrjournals.org/content/77/4 _ Supplement/P6-11-04.

62. Butler CC, Coenen S, Saville BR, Cook J, van der Velden A, Homes J, et al. A trial like ALIC4E: Why design a platform, response-adaptive, open, randomised controlled trial of antivirals for influenza-like illness? ERJ Open Res. 2018;4:00046-2018. 
63. Wen PY, Alfred Yung WK, David Adelson P, Alexander BM, Alper J, Arnold $\mathrm{MM}$, et al. Adaptive global innovative learning environment for glioblastoma: GBM AGILE. Clin Cancer Res. 2018;24:737-43.

64. Diamond EL, Subbiah V, Craig Lockhart A, Blay JY, Puzanov I, Chau I, et al. Vemurafenib for BRAF V600-mutant erdheim-chester disease and langerhans cell histiocytosis analysis of data from the histologyindependent, phase 2, open-label VE-BASKET study. JAMA Oncol. 2018;4: 384-8.

65. Hyman DM, Puzanov I, Subbiah V, Faris JE, Chau I, Blay JY, et al.

Vemurafenib in Multiple Nonmelanoma Cancers with BRAF V600 Mutations. N Engl J Med. 2015;373:726-36.

\section{Publisher's Note}

Springer Nature remains neutral with regard to jurisdictional claims in published maps and institutional affiliations.

Ready to submit your research? Choose BMC and benefit from:

- fast, convenient online submission

- thorough peer review by experienced researchers in your field

- rapid publication on acceptance

- support for research data, including large and complex data types

- gold Open Access which fosters wider collaboration and increased citations

- maximum visibility for your research: over $100 \mathrm{M}$ website views per year

At BMC, research is always in progress.

Learn more biomedcentral.com/submissions 